\title{
Quasiparticle Swarm Optimization for Cross-Section Linear Profile Error Evaluation of Variation Elliptical Piston Skirt
}

\author{
Xiulan Wen, Yibing Zhao, Youxiong Xu, and Danghong Sheng \\ Automation Department, Nanjing Institute of Technology, Nanjing 211167, China \\ Correspondence should be addressed to Xiulan Wen, zdhxwxl@njit.edu.cn
}

Received 28 August 2011; Revised 23 October 2011; Accepted 14 November 2011

Academic Editor: Andrzej Swierniak

Copyright (C) 2012 Xiulan Wen et al. This is an open access article distributed under the Creative Commons Attribution License, which permits unrestricted use, distribution, and reproduction in any medium, provided the original work is properly cited.

Variation elliptical piston skirt has better mechanical and thermodynamic properties and it is widely applied in internal combustion engine in recent years. Because of its complex form, its geometrical precision evaluation is a difficult problem. In this paper, quasi-particle swarm optimization (QPSO) is proposed to calculate the minimum zone error and ellipticity of crosssection linear profile, where initial positions and initial velocities of all particles are generated by using quasi-random Halton sequences which sample points have good distribution properties and the particles' velocities are modified by constriction factor approach. Then, the design formula and mathematical model of the cross-section linear profile of variation elliptical piston skirt are set up and its objective function calculation approach using QPSO to solve the minimum zone crosssection linear profile error is developed which conforms to the ISO/1101 standard. Finally, the experimental results evaluated by QPSO, particle swarm optimization (PSO), improved genetic algorithm (IGA) and the least square method (LSM) confirm the effectiveness of the proposed QPSO and it improves the linear profile error evaluation accuracy and efficiency. This method can be extended to other complex curve form error evaluation such as cam curve profile.

\section{Introduction}

Piston skirt is the key parts of internal combustion engines (ICEs). Because internal combustion engines usually run under the circumstance of higher speed, larger pressure, and heavier load, it makes piston skirt work in overload conditions. Besides, piston skirt is also an important source of lubrication failures that will in turn lead to noise and power loss arisen from friction forces [1]. Therefore, it is necessary to improve its design, manufacture, measurement and evaluation method. With the rapid development of ICE industry, higher and higher design requirement for piston skirt is proposed for realizing high speed, high efficiency, low consumption, and low noise. The piston skirts of traditional formal cylinder 
and formal cone are seldom used, and they are mostly replaced with complex newstyle profile piston skirts, especially for automobile engine. Among them convex variation elliptical piston skirt is widely applied that has the characteristics that the cross-section profiles at different heights are different ellipses and the axis-section profile is a convex curve. Compared with traditional one, the convex variation elliptical piston skirt has much better mechanical and thermodynamic properties. But the geometrical precision evaluation is a difficult problem because of its complex form. Recently many researchers have devoted themselves to develop different algorithms to compute the cross-section linear profile error of piston skirt. Huang et al. [2] proposed the algorithm based on single optimum seeking and moment method to evaluate cross-section linear profile error. In the hypothesis conditions of small deviation and small error with the measured data, least square cross-section linear profile error was calculated. Based on the combination of moment method and least square method, Huang and Wang [3] also put forward the evaluation method of piston skirt crosssection linear profile error. Liu et al. [4] set up the mathematical model of cross-section linear profile and deduced relevant designing formulae. The least square cross-section linear profile error was calculated. Nowadays most algorithms are based on LSM because of its ease of computation and the uniqueness of its solution. Because ISO 1101 (1996) recommends the form tolerance being evaluated based on the concept of minimum zone [5], the minimum zone method (MZM) has received much attention in recent years, and it has been applied to solve circularity (roundness), straightness, flatness, sphericity, and cylindricity error. Cheraghi et al. [6] formulated straightness and flatness errors by nonlinear optimization problems with linear objective function and nonlinear constraints. Samuel and Shunmugam [7] established the minimum circumscribed limacoid, maximum inscribed limacoid, and minimum zone limacoid in literature based on the computational geometry to evaluate sphericity error from coordinate measurement data. Weber et al. [8] proposed a unified linear approximation technique for use in evaluating the forms of straightness, flatness, circularity, and cylindricity. Non-linear equation for each form was linearized using Taylor expansion, and then it was solved as a linear program. Zhu and Ding [9] established the equivalence between the width of a point set and the inner radius of the convex hull of the selfdifference of the set. An algorithm was proposed to calculate the "almost exact" minimum zone solution, which is implemented by solving a single linear programming problem. Li and Shi [10] applied the curvature technique for solving problems of roundness evaluation from coordinate data measured by CMM. Above methods are effective in solving simple form errors such as straightness, flatness, circularity, sphericity and cylindricity. Because the cross-section linear profile of variation elliptical piston skirt is more complex, it is difficult for traditional method to calculate the minimum zone error.

With the emergence of computational intelligence, the intelligence-oriented algorithms such as genetic algorithms (GAs) and particle swarm optimization (PSO) have been successfully employed to evaluate form error such as flatness, straightness, cylindricity, and so forth [11-13]. Wen attempted to calculate the minimum zone solution of piston skirt cross-section linear profile error by PSO [14]. Because there are several approximations in establishing profile error mathematical model, the solution is not very accurate.

In order to solve the minimum zone error of piston skirt cross-section linear profile accurately and efficiently, its mathematical model is founded, and QPSO is proposed. The paper is organized as follows: the design formula and mathematical model of the crosssection linear profile of variation elliptical piston skirt are set up. Then, QPSO for piston skirt cross-section linear profile error evaluation is developed. Finally, the examples are given, and conclusions are drawn. 


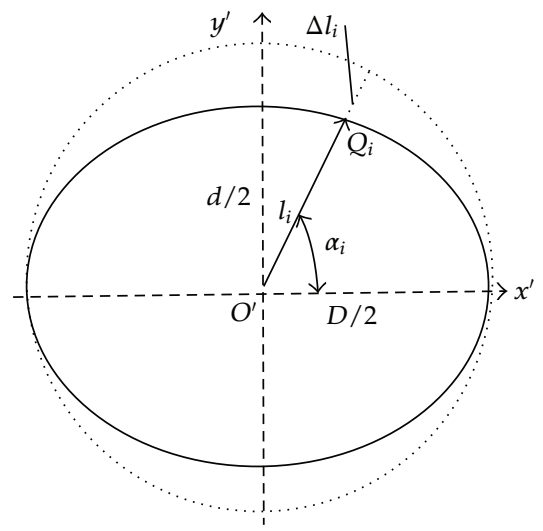

Figure 1: The cross-section profile of variation elliptical piston skirt.

\section{Mathematical Model of Piston Skirt Cross-Section Linear Profile Error Evaluation}

\subsection{Design Formula}

Set up the design coordinate system $x^{\prime} O^{\prime} y^{\prime}$ of the cross-section linear profile of variation elliptical piston skirt, shown in Figure 1. $Q_{i}$ is the design point on the cross-section linear profile. The radial reduction $\Delta l_{i}$ of the point $Q_{i}$ is usually formulated as [4]

$$
\Delta l_{i}=\frac{D-d}{4}\left[\left(1-\cos 2 \alpha_{i}\right)+\frac{b}{25}\left(1-\cos 4 \alpha_{i}\right)\right],
$$

where $D$ is the diameter of long axis, $d$ is the diameter of short axis, $\alpha_{i}$ is the polar angle of the point $Q_{i}, b$ is the coefficient of plump degree, and $G=D-d$ is the ellipticity.

The design formula of the point $Q_{i}$ on cross-section linear profile is formulated as

$$
l_{i}=\frac{D}{2}-\Delta l_{i}=\frac{D}{2}-\frac{D-d}{4}\left[\left(1-\cos 2 \alpha_{i}\right)+\frac{b}{25}\left(1-\cos 4 \alpha_{i}\right)\right],
$$

where $l_{i}$ is the radius of the point $Q_{i}$.

\subsection{Mathematics Model of Cross-Section Linear Profile}

The measurement model of cross-section linear profile of variation elliptical piston skirt is shown in Figure 2.

$O$ is the revolving centre of the measurement platform and $O^{\prime}$ is the design center of piston skirt, $e$ is the setting eccentricity $\left(e=O O^{\prime}\right)$ and $\theta_{0}$ is the eccentric angle, and $\phi_{0}$ is the angle between the measurement coordinate axis $O x$ and the long axis $O^{\prime} x^{\prime}$ of design profile $\left(-10^{\circ} \leq \phi_{0} \leq 10^{\circ}\right)$. Assuming that $P_{i}\left(r_{i}, \theta_{i}\right)(i=1,2, \ldots, n, n$ is the number of measured point) is the measured point of the cross-section linear profile corresponding to the revolving centre $O$, and $r_{i}$ and $\theta_{i}$ are the radius and polar angle of point $P_{i}$, respectively. $P_{i}^{\prime}\left(r_{i}^{\prime}, \theta_{\mathrm{i}}^{\prime}\right)$ is the mapping point of $P_{i}$, and $r_{i}^{\prime}$ and $\theta_{i}^{\prime}$ are the radius and polar angle of point $P_{i}^{\prime}$ in the design 


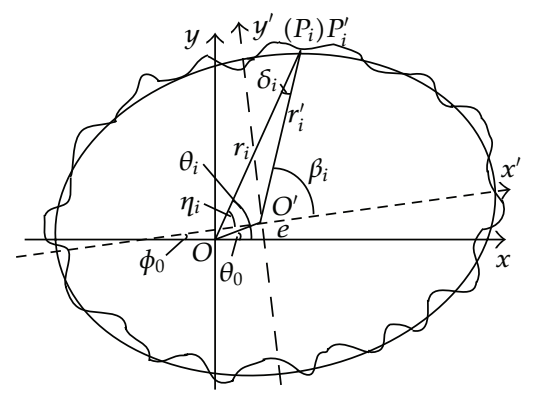

Figure 2: The measurement model of cross-section profile.

coordinate system $x^{\prime} O^{\prime} y^{\prime} . \beta_{i}$ is the angle between $P_{i}^{\prime} O^{\prime}$ and $O^{\prime} x^{\prime}, \delta_{i}$ is the angle between $O P_{i}^{\prime}$ and $O^{\prime} P_{i}^{\prime}$. Because the setting eccentricity $e$ is very small, and $\delta_{i}$ is also very small.

Using cosine theorem in the triangle $\Delta P_{i}^{\prime} O O^{\prime}$, we get the following:

$$
r_{i}^{\prime 2}=r_{i}^{2}+e^{2}-2 e r_{i} \cos \left(\theta_{i}-\theta_{0}\right)
$$

Equation (2.3) can be rewritten

$$
r_{i}^{\prime}=\sqrt{r_{i}^{2}+e^{2}-2 e r_{i} \cos \left(\theta_{i}-\theta_{0}\right)} .
$$

From Figure 2, we can learn

$$
\begin{aligned}
& \beta_{i}=\delta_{i}+\eta_{i}, \\
& \theta_{i}=\eta_{i}+\phi_{0} .
\end{aligned}
$$

So, we get the following:

$$
\beta_{i}=\theta_{i}-\phi_{0}+\delta_{i}
$$

According to Taylor series expansion, we have the following

$$
\begin{aligned}
& \cos 2 \beta_{i}=\cos 2\left(\theta_{i}-\phi_{0}+\delta_{i}\right) \approx \cos 2\left(\theta_{i}-\phi_{0}\right)-2 \sin 2\left(\theta_{i}-\phi_{0}\right) \cdot \delta_{i} \\
& \cos 4 \beta_{i}=\cos 4\left(\theta_{i}-\phi_{0}+\delta_{i}\right) \approx \cos 4\left(\theta_{i}-\phi_{0}\right)-4 \sin 4\left(\theta_{i}-\phi_{0}\right) \cdot \delta_{i} .
\end{aligned}
$$

When $\beta_{i}=\alpha_{i}$, the radius design value $l_{i}$ corresponding to the polar $\alpha_{i}$ can be rewritten

$$
\begin{aligned}
l_{i}=\frac{D}{2}-\frac{D-d}{4}[1 & -\cos 2\left(\theta_{i}-\phi_{0}\right)+2 \sin 2\left(\theta_{i}-\phi_{0}\right) \cdot \delta_{i} \\
& \left.+\frac{b}{25}\left(1-\cos 4\left(\theta_{i}-\phi_{0}\right)+4 \sin 4\left(\theta_{i}-\phi_{0}\right) \cdot \delta_{i}\right)\right] .
\end{aligned}
$$


Using sine theorem in the triangle $\Delta P_{i}^{\prime} O O^{\prime}$, we get the following

$$
\frac{e}{\sin \delta_{i}}=\frac{r_{i}^{\prime}}{\sin \left(\theta_{i}-\theta_{0}\right)}
$$

Because $\delta_{i}$ is very small, $\delta_{i} \approx \sin \delta_{i}$, and it is substituted into (2.9), then (2.9) can be approximated as

$$
\delta_{i}=\frac{e \sin \left(\theta_{i}-\theta_{0}\right)}{r_{i}^{\prime}}
$$

Substituting (2.10) into (2.8), we have the following:

$$
\begin{aligned}
l_{i}=\frac{D}{2}-\frac{D-d}{4}[ & 1-\cos 2\left(\theta_{i}-\phi_{0}\right)+\frac{2 \sin 2\left(\theta_{i}-\phi_{0}\right) \cdot e \sin \left(\theta_{i}-\theta_{0}\right)}{r_{i}^{\prime}} \\
& \left.+\frac{b}{25}\left(1-\cos 4\left(\theta_{i}-\phi_{0}\right)+\frac{4 \sin 4\left(\theta_{i}-\phi_{0}\right) \cdot e \sin \left(\theta_{i}-\theta_{0}\right)}{r_{i}^{\prime}}\right)\right] .
\end{aligned}
$$

\subsection{The Objective Function in Using QPSO to Calculate the Minimum Zone Error}

The deviation $\varepsilon_{i}$ between the polar radius $r_{i}^{\prime}$ of the mapping point $P_{i}^{\prime}$ and the polar radius $l_{i}$ of the design point $Q_{i}$ corresponding to the same polar angle is

$$
\begin{aligned}
\varepsilon_{i}=r_{i}^{\prime}-l_{i} & \\
=r_{i}^{\prime}-\left\{\frac{D}{2}-\frac{D-d}{4}[\right. & 1-\cos 2\left(\theta_{i}-\phi_{0}\right)+\frac{2 \sin 2\left(\theta_{i}-\phi_{0}\right) \cdot e \sin \left(\theta_{i}-\theta_{0}\right)}{r_{i}^{\prime}} \\
& \left.\left.+\frac{b}{25}\left(1-\cos 4\left(\theta_{i}-\phi_{0}\right)+\frac{4 \sin 4\left(\theta_{i}-\phi_{0}\right) \cdot e \sin \left(\theta_{i}-\theta_{0}\right)}{r_{i}^{\prime}}\right)\right]\right\},
\end{aligned}
$$

where $r_{i}^{\prime}=\sqrt{r_{i}^{2}+e^{2}-2 e r_{i} \cos \left(\theta_{i}-\theta_{0}\right)}$.

According to the ISO/1101 standard, the minimum zone solution of linear profile error is the minimum width value of two ideal equidistance design profiles which encompass the measured real profile. Therefore, the objective function in using QPSO to calculate the minimum zone error of cross-section linear profile can be expressed as:

$$
f\left(\theta_{0}, e, \phi_{0}, \beta, D, d\right)=\min \left(\max \left(\varepsilon_{i}\right)-\min \left(\varepsilon_{i}\right)\right) .
$$

Equation (2.13) is a function of $\left(\theta_{0}, e, \phi_{0}, \beta, D, d\right)$. Consequently, solving the minimum zone cross-section linear profile error of variation elliptical piston skirt is translated into searching the values of the parameters set $\left(\theta_{0}, e, \phi_{0}, \beta, D, d\right)$, so that the objective function $f\left(\theta_{0}, e, \phi_{0}, \beta, D, d\right)$ is the minimum. 


\section{Using QPSO to Evaluate Piston Skirt Cross-Section Linear Profile Error}

\subsection{Pseudorandom Numbers and Quasirandom Halton Sequences}

\subsubsection{Pseudorandom Numbers and Quasirandom Sequences}

Pseudorandom numbers are deterministic, but they try to imitate an independent sequence of genuine random numbers. Common pseudorandom number generators include linear congruential, quadratic congruential, inversive congruential, parallel linear congruential, et.al. In contrast to pseudorandom numbers, the points in a quasirandom sequence do not imitate genuine random points. But they try to cover the feasible region in an optimal way. Quasirandom generators do not generate numbers, but sequences of points in the desired dimension. Common quasirandom sequence generators include Halton, Hammersley, Faure, Sobol, and Niederreiter generators [15]. In this paper we focus our attention on Halton sequence since it is conceptually very appealing, and it can be produced easily and fast with simple algorithms.

\subsubsection{Halton Sequences}

Halton sequences are not unique, and they depend on the set of prime numbers taken as bases to construct their vector components. Typically and most efficiently, the lowest possible primes are used. tation as

Let $b$ be a prime number. Then any integer $k, k \geq 0$, can be written in base- $b$ represen-

$$
k=d_{j} b^{j}+d_{j-1} b^{j-1}+\cdots+d_{1} b+d_{0}
$$

where $d_{i} \in\{0,1, \ldots, b-1\}, i=0,1, \ldots, j$. Define the base- $b$ radical inverse function, $\phi_{b}(k)$, as

$$
\phi_{b}(k)=\frac{d_{0}}{b^{1}}+\frac{d_{1}}{b^{2}}+\cdots+\frac{d_{j}}{b^{j+1}} .
$$

Notice that for every integer, $k \geq 0, \phi_{b}(k) \in[0,1]$.

The $k$ th element of the Halton sequence is obtained via the radical inverse function evaluated at $k$. Specifically, if $b_{1}, \ldots, b_{d}$ are $d$ different prime numbers, then a $d$-dimensional Halton sequence of length $m$ is given by $\left\{x_{1}, \ldots, x_{m}\right\}$, where the $k$ th element of the sequence is

$$
X_{k}=\left[\phi_{b_{1}}(k-1), \ldots, \phi_{b_{d}}(k-1)\right]^{T}, \quad k=1, \ldots, m
$$

\subsection{QPSO for Evaluating Cross-Section Linear Profile Error of Piston Skirt}

Particle swarm optimization (PSO) method is one of the most powerful methods for solving unconstrained and constrained global optimization problems. The method was originally proposed by Kennedy and Eberhart as an optimization method in 1995 [16], which was inspired by the social behavior of bird flocking and fish schooling. It utilizes a "population" of particles that fly through the problem hyperspace with given velocities [17]. In PSO 
initial position and initial velocity of particles are often randomly generated by using pseudorandom numbers $[13,18]$. Because the positions of initial particles have influence on the optimization performance, Richard and Ventura [19] proposed initializing the particles in a way that they are distributed as evenly as possible throughout the problem space. This ensures a broad coverage of the search space. They concluded that applying a starting configuration based on the centroidal Voronoi tessellations (CVTs) improves the performance of the PSO compared with the original random initialization. As an alternative method, Campana et al. [20] proposed reformulating the standard iteration of PSO into a linear dynamic system. The system can then be investigated to determine the initial particles' positions such that the trajectories over the problem hyperspace are orthogonal, improving the exploration mode and convergence of the swarm.

Quasirandom sequences have been successfully applied in numerical integration and in random search optimization methods [21]. The idea of a good initial population has also been used in genetic programming and genetic algorithm [22]. In this work, quasirandom Halton sequences are applied to generate the initial positions and velocities of particles in PSO for solving the minimum zone profile error of variation elliptical piston skirts. For short, the proposed PSO is called quasiparticle swarm optimization (QPSO).

QPSO algorithm begins by using quasirandom Halton sequences to initialize a swarm of $N$ particles ( $N$ is referred as particle size), each having $s$ unknown parameters ( $s$ is referred as the dimensionality of optimized variables) to be optimized at each iteration. The ideal cross-section linear profile can be decided by the set of six parameters $\left(\theta_{0}, e, \phi_{0}, \beta, D, d\right)$ and the method takes $\left(\theta_{0}, e, \phi_{0}, \beta, D, d\right)$ as a particle. Therefore, the dimension $s$ of the particle is six. The best particle with the minimum objective function value $f\left(\theta_{0}, e, \phi_{0}, \beta, D, d\right)$ is considered as the minimum zone solution to the cross-section linear profile error. The flow of QPSO for evaluating cross-section linear profile error is as follows.

Step 1. Input the measurement values $\left(r_{i}, \theta_{i}\right)(i=1,2, \ldots, n)$ of the cross-section linear profile. If the point is measured in the Cartesian coordinates, it needs to be transformed into the polar coordinates.

Step 2. Generate the initial positions and initial velocities of all particles by using quasirandom Halton sequences.

Step 3. Calculate the objective functions of all particles according to (2.13). The less the objective function value is, the better the particle is.

Step 4. Update velocity. Because constriction factor approach (CFA) ensures the convergence of the search procedures based on the mathematical theory and can generate higher-quality solutions [23], CFA is employed to modify the velocity. The velocity and position parameters of each particle $\left(p_{i}\right)$ in the swarm are updated at iteration $(t)$ according to CFA:

$$
\begin{gathered}
v_{i}^{t+1}=K\left(v_{i}^{t}+c_{1} r_{1 j}\left(\text { pbest } t_{i}^{t}-p_{i}^{t}\right)+c_{2} r_{2 j}\left(\text { gbest }^{t}-p_{i}^{t}\right)\right), \\
K=\frac{2}{\left|2-\varphi-\sqrt{\varphi^{2}-4 \varphi}\right|}
\end{gathered}
$$

where $v_{i}^{t}$ and $p_{i}^{t}$ are the velocity and position of $i$ th particle at iteration $t$, respectively. $r_{1 j}$ and $r_{2 j}(j=1,2, \ldots, s)$ are uniform random numbers between 0 and $1 . c_{1}$, and $c_{2}$ are acceleration 
factors that determine the relative pull for each particle toward its previous best position (pbest) and the group's best position (gbest), respectively. $c_{1}$ and $c_{2}$ meet the conditions $\varphi=$ $c_{1}+c_{2}$.

Step 5. Update position. The position of each particle is modified by

$$
p_{i}^{t+1}=p_{i}^{t}+v_{i}^{t} \Delta t
$$

Step 6. Update pbest. Calculate the objective function of all particles. If the current objective function value of a particle is less than the old pbest value, the pbest is replaced with the current position.

Step 7. Update gbest. If the current objective function value of a particle is less than the old gbest value, the gbest is replaced with the current position.

Step 8. Go to Step 4 until the maximum iteration is satisfied.

Step 9. Output the computation results.

\section{Results and Discussion}

\subsection{Optimizing Classical Testing Functions}

In order to verify the optimization efficiency of QPSO, numerical experiments on some classical testing functions are carried out [24]. Two examples are given as follows.

\section{Function 1}

It is the Rosenbrock function defined

$$
f_{1}(x)=100 \times\left(x_{2}-x_{1}^{2}\right)^{2}+\left(1-x_{1}\right)^{2}, \quad-2.048 \leq x_{1}, x_{2} \leq 2.048
$$

It is hard to be minimized. The global minimum point is at $(1.0,1.0)$, and the global minimum is zero.

\section{Function 2}

It is the Schaffer test function defined as

$$
f_{2}(x)=0.5+\frac{\sin ^{2} \sqrt{x_{1}^{2}+x_{2}^{2}}-0.5}{\left[1.0+0.001 \times\left(x_{1}^{2}+x_{2}^{2}\right)\right]^{2}}, \quad-100 \leq x_{i} \leq 100 .
$$

This function has many circle ridges nearby the global minimum (1.0, 1.0), and the function value of the nearest circle ridge $\left(x_{1}^{2}+x_{2}^{2}=3.138^{2}\right)$ is 0.009716 . It is very easy to trap in this value.

The proposed algorithms were written in MATLAB, and the experiments were run in Windows XP on an IBM ThinkPad X200-7457 A46 with 2.26 GHz main frequency and 1GB 
Table 1: Mean and standard deviation of functions.

\begin{tabular}{lcccc}
\hline Fun. & Parameters & \multicolumn{3}{c}{ Methods } \\
& & QPSO & PSO & IGA \\
\hline \multirow{2}{*}{ Fun. 1 } & Mean & $1.2458 e-10$ & $1.6208 e-9$ & $6.8649 e-4$ \\
& Standard dev & $3.0030 e-10$ & $5.6145 e-9$ & $1.0317 e-3$ \\
Fun. 2 & Mean & 0.0019 & 0.0036 & 0.0073 \\
& Standard dev & 0.0040 & 0.0051 & 0.0035 \\
\hline
\end{tabular}

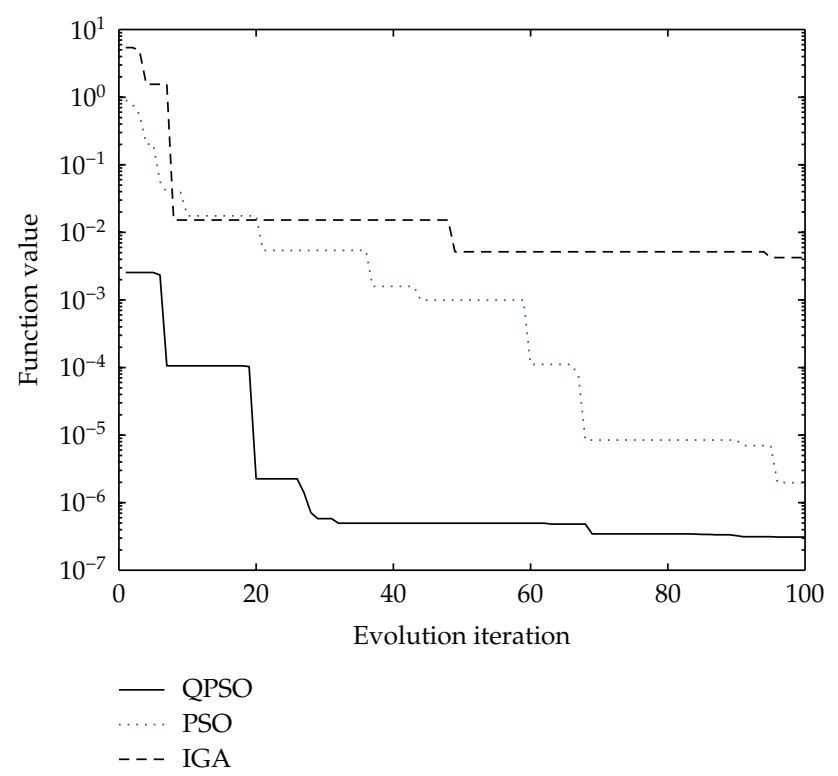

Figure 3: The evolution process of function $f_{1}$ by three different methods.

memory. QPSO is also a stochastic optimization method and it is important to evaluate the average performance. For comparison, two stochastic optimization methods including PSO [13] and IGA [11] are employed. The popsize size was set 50, and 20 trials were performed in prescribed maximum iteration 200. In specified initial ranges, initial populations and initial positions were randomly generated by using pseudorandom numbers for PSO and IGA. Initial populations were generated by using Halton random sequences for QPSO. The mean values and the standard deviations are tabulated in Table 1.

As seen in Table 1, the QPSO method for two examples could provide more accurate and stable solution. Figures 3 and 4 show the optimizing processes of these methods at one trial for two examples, respectively. As seen in the figures, it is evident that the optimization performance of QPSO is better than those of PSO and IGA.

\subsection{Examples}

\section{Simulation Example}

According to the design formula of cross-section linear profile of variation elliptical piston skirt, the simulation data with random noise are generated. The setting eccentricity and the 


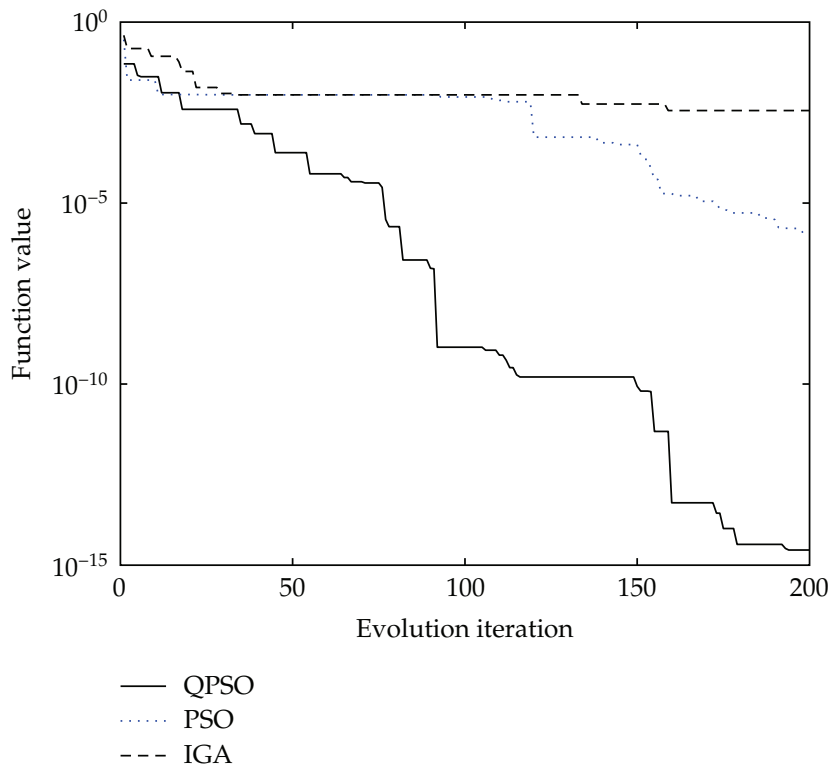

Figure 4: The evolution process of function $f_{2}$ by three different methods.

Table 2: Results of simulate example.

\begin{tabular}{lcccc}
\hline Parameters & Design value & $\begin{array}{c}\text { Results by } \\
\text { QPSO }\end{array}$ & Results by PSO & Results by IGA \\
\hline$D$ & 46 & 45.9814 & 46.0846 & 45.9298 \\
$d$ & 45 & 44.9715 & 45.0779 & 44.9216 \\
$\beta$ & 0.62 & 0.6636 & 0.5953 & 0.2429 \\
$e$ & 0.01 & 0.0124 & 0.0138 & 0.0110 \\
$\theta_{0}$ & 0.1 & 0.2048 & 0.1157 & -0.0485 \\
$\varphi_{0}$ & 0.05 & 0.0471 & 0.0438 & 0.0462 \\
Contour error & 0.0774 & 0.0775 & 0.0779 & 0.0797 \\
\hline
\end{tabular}

The unit of length is $\mathrm{mm}$, and the unit of angle is radian.

angle between the measurement coordinate axis and the long axis of design profile are set by the coordinate translation and rotation transform. In the experiment, the design data of $\theta_{0}, e, \phi_{0}, \beta, D, d$ are listed in Table 2 , and the transformed simulation data are shown in Table 3.

For comparison, IGA and PSO were employed. Considering the values of optimized parameters, $\theta_{0}, e, \phi_{0}$, and $\beta$ are very small, and $D$ and $d$ are usually larger, in order to save searching time, the initial populations and initial positions were randomly generated by using pseudorandom numbers in: $\left([-\varepsilon, \varepsilon],[-\varepsilon, \varepsilon],[-\varepsilon, \varepsilon],[-\varepsilon, \varepsilon],\left[-\varepsilon+\max \left(r_{i}\right), \varepsilon+\max \left(r_{i}\right)\right],[-\varepsilon+\right.$ $\left.\left.\min \left(r_{i}\right), \varepsilon+\min \left(r_{i}\right)\right]\right)$ for IGA and PSO. For QPSO the initial positions were generated by using quasrandom Halton sequence in $\left([-\varepsilon, \varepsilon],[-\varepsilon, \varepsilon],[-\varepsilon, \varepsilon],[-\varepsilon, \varepsilon],\left[-\varepsilon+\max \left(r_{i}\right), \varepsilon+\max \left(r_{i}\right)\right],[-\varepsilon+\right.$ $\left.\left.\min \left(r_{i}\right), \varepsilon+\min \left(r_{i}\right)\right]\right)$ and the initial velocities were generated by using quasirandom Halton sequence in: $([-\varepsilon, \varepsilon],[-\varepsilon, \varepsilon],[-\varepsilon, \varepsilon],[-\varepsilon, \varepsilon],[-\varepsilon, \varepsilon],[-\varepsilon, \varepsilon])$. In our experiments, $\varepsilon$ is all set 0.5 . The searching process and optimization results of the minimum zone error of crosssection linear profile by different methods are shown in Figure 5 and Table 2. As seen in Figure 5 and Table 2, the minimum zone error by QPSO is $0.0775 \mathrm{~mm}$ and it is smaller than 
Table 3: Simulated measurement data.

\begin{tabular}{|c|c|c|}
\hline No & $r_{i}$ & $\theta_{i}$ \\
\hline 1 & 23.0278 & 2.8649 \\
\hline 2 & 23.0024 & 7.8669 \\
\hline 3 & 22.9572 & 12.8633 \\
\hline 4 & 22.9367 & 17.8596 \\
\hline 5 & 22.9843 & 22.8560 \\
\hline 6 & 22.9393 & 27.8580 \\
\hline 7 & 22.8743 & 32.8544 \\
\hline 8 & 22.8715 & 37.8507 \\
\hline 9 & 22.7498 & 42.8528 \\
\hline 10 & 22.7478 & 47.8491 \\
\hline 11 & 22.7198 & 52.8455 \\
\hline 12 & 22.6964 & 57.8475 \\
\hline 13 & 22.6195 & 62.8439 \\
\hline 14 & 22.6054 & 67.8459 \\
\hline 15 & 22.5901 & 72.8423 \\
\hline 16 & 22.552 & 77.8443 \\
\hline 17 & 22.485 & 82.8407 \\
\hline 18 & 22.5278 & 87.8428 \\
\hline 19 & 22.4691 & 92.8448 \\
\hline 20 & 22.5276 & 97.8412 \\
\hline 21 & 22.5447 & 102.8432 \\
\hline 22 & 22.5262 & 107.8453 \\
\hline 23 & 22.5110 & 112.8416 \\
\hline 24 & 22.5500 & 117.8437 \\
\hline 25 & 22.5974 & 122.8458 \\
\hline 26 & 22.6383 & 127.8478 \\
\hline 27 & 22.7235 & 132.8499 \\
\hline 28 & 22.7497 & 137.8520 \\
\hline 29 & 22.8111 & 142.8541 \\
\hline 30 & 22.8098 & 147.8561 \\
\hline 31 & 22.8188 & 152.8582 \\
\hline 32 & 22.8955 & 157.8603 \\
\hline 33 & 22.8889 & 162.8623 \\
\hline 34 & 22.9762 & 167.8644 \\
\hline 35 & 22.9514 & 172.8665 \\
\hline 36 & 22.9563 & 177.8685 \\
\hline 37 & 22.9827 & 182.8706 \\
\hline 38 & 23.0158 & 187.8727 \\
\hline 39 & 22.9803 & 192.8747 \\
\hline 40 & 22.9512 & 197.8768 \\
\hline 41 & 22.8895 & 202.8789 \\
\hline 42 & 22.9254 & 207.8809 \\
\hline 43 & 22.8449 & 212.8830 \\
\hline 44 & 22.845 & 217.8851 \\
\hline 45 & 22.7707 & 222.8872 \\
\hline 46 & 22.7421 & 227.8892 \\
\hline
\end{tabular}


Table 3: Continued.

\begin{tabular}{|c|c|c|}
\hline No & $r_{i}$ & $\theta_{i}$ \\
\hline 47 & 22.6823 & 232.8913 \\
\hline 48 & 22.6402 & 237.8934 \\
\hline 49 & 22.5739 & 242.8954 \\
\hline 50 & 22.5962 & 247.8918 \\
\hline 51 & 22.5604 & 252.8938 \\
\hline 52 & 22.5023 & 257.8959 \\
\hline 53 & 22.5435 & 262.8980 \\
\hline 54 & 22.4712 & 267.9000 \\
\hline 55 & 22.4814 & 272.8964 \\
\hline 56 & 22.5042 & 277.8985 \\
\hline 57 & 22.4754 & 282.9005 \\
\hline 58 & 22.5467 & 287.8969 \\
\hline 59 & 22.5529 & 292.8989 \\
\hline 60 & 22.5736 & 297.8953 \\
\hline 61 & 22.5889 & 302.8973 \\
\hline 62 & 22.6577 & 307.8937 \\
\hline 63 & 22.6912 & 312.8958 \\
\hline 64 & 22.7493 & 317.8921 \\
\hline 65 & 22.7944 & 322.8942 \\
\hline 66 & 22.8161 & 327.8905 \\
\hline 67 & 22.8911 & 332.8868 \\
\hline 68 & 22.9278 & 337.8889 \\
\hline 69 & 22.9161 & 342.8852 \\
\hline 70 & 22.9957 & 347.8816 \\
\hline 71 & 23.0318 & 352.8779 \\
\hline 72 & 23.0318 & 357.8800 \\
\hline
\end{tabular}

$r_{i}: \mathrm{mm}, \theta_{i}:$ degree.

that by PSO and IGA. It takes about 40 iterations for the proposed QPSO to find the optimal solution and it is faster than PSO and IGA. The cross-section linear profile error by LSM is $0.0932 \mathrm{~mm}$ and it is larger than the minimum zone error.

\section{Practical Example}

The cross-section profiles of piston skirt of a SL 105 diesel engine are variation ellipses and its main cross-section profiles (upper end, convexity and lower end) are inspected by Coordinate Measurement Machine (CMM). And the minimum zone error of every cross-section linear profile is calculated by the proposed QPSO and the results are listed in Table 4. For comparison, the ellipticities of three cross-sections are calibrated by 19JPC microcomputer-type all-purpose tool microscope and the values are also listed in Table 4. From the table, we can learn the cross-section linear profile error of MZM is less than that of LSM. And the ellipticity calculated by QPSO is almost the same as the calibration value. 


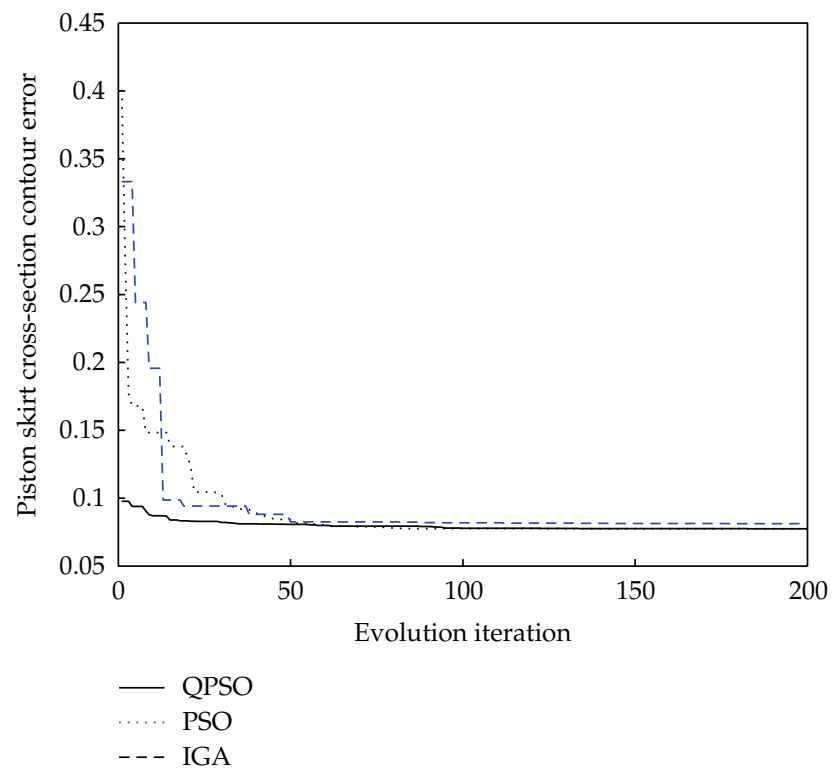

Figure 5: The evolution process by different methods.

Table 4: Results of practical example.

\begin{tabular}{lcccc}
\hline $\begin{array}{l}\text { Cross-section } \\
\text { positions }\end{array}$ & $\begin{array}{c}\text { The linear profile } \\
\text { error of MZM }\end{array}$ & $\begin{array}{c}\text { The linear profile } \\
\text { error of LSM }\end{array}$ & $\begin{array}{c}\text { Calibration value of } \\
\text { ellipticity }\end{array}$ & $\begin{array}{c}\text { Ellipticity calculated } \\
\text { by QPSO }\end{array}$ \\
\hline Upper end & 0.1023 & 0.1225 & 0.0251 & 0.0252 \\
Convexity & 0.0984 & 0.1189 & 0.0210 & 0.0208 \\
Lower end & 0.0912 & 0.0996 & 0.0181 & 0.0179 \\
\hline
\end{tabular}

Unit: $\mathrm{mm}$

\section{Conclusions}

In this paper, QPSO is proposed to calculate the minimum zone error and ellipticity of cross-section linear profile of variation elliptical piston skirt, which initial positions and initial velocities of all particles are generated by using quasirandom Halton sequences and the particles' velocities are modified by constriction factor approach. The design formula and mathematical model of the cross-section linear profile are set up and its objective function calculation approach using QPSO to solve the minimum zone error of cross-section linear profile is developed. The simulation and practical examples confirm the optimization efficiency of QPSO is better than that of PSO and IGA for complex optimal problems. Compared with conventional evaluation methods, the proposed method not only has the advantages of simple algorithm and good flexibility, but also improves cross-section linear profile error evaluation accuracy. The proposed method can be extended to other complex curve profile error evaluation.

\section{Acknowledgments}

The research was supported by Natural Science Research Project of the People's Republic of China (no: 51075198), Natural Science Research Project of Jiangsu Province (BK2010479) 
Jiangsu Provincial Project of 333 Talents Engineering of China, Jiangsu Provincial Project of Six Talented Peak of China.

\section{References}

[1] F. M. Meng, Y. Y. Zhang, Y. Z. Hu, and H. Wang, "Thermo-elasto-hydrodynamic lubrication analysis of piston skirt considering oil film inertia effect," Tribology International, vol. 40, no. 7, pp. 1089-1099, 2007.

[2] R. Y. Huang, N. X. Wang, and Q. Yang, “The evaluation of piston skirt contour error," Acta Northwest Agriculture University, vol. 21, no. 3, pp. 41-46, 1993 (Chinese).

[3] R. Y. Huang and N. X. Wang, "Study on the evaluation of piston skirt cross-section contour error with moment method," Transactions of CSICE, vol. 14, no. 1, pp. 84-91, 1996 (Chinese).

[4] H. G. Liu, D. A. Wan, X. L. Min et al., "Study on least square method about the evaluation of contour of cross section of variation elliptical piston skirt," Journal of Tongji University, vol. 28, no. 2, pp. 231-235, 2000 (Chinese).

[5] ISO/DIS 1101-1996, Technical drawings-geometrical tolerancing, ISO, Geneva, Switzerland, 1996.

[6] S. H. Cheraghi, H. S. Lim, and S. Motavalli, "Straightness and flatness tolerance evaluation: an optimization approach," Precision Engineering, vol. 18, no. 1, pp. 30-37, 1996.

[7] G. L. Samuel and M. S. Shunmugam, "Evaluation of sphericity error from coordinate measurement data using computational geometric techniques," Computer Methods in Applied Mechanics and Engineering, vol. 190, no. 51-52, pp. 6765-6781, 2001.

[8] T. Weber, S. Motavalli, B. Fallahi, and S. H. Cheraghi, "A unified approach to form error evaluation," Precision Engineering, vol. 26, no. 3, pp. 269-278, 2002.

[9] X. Zhu and H. Ding, "Flatness tolerance evaluation: an approximate minimum zone solution," CAD Computer Aided Design, vol. 34, no. 9, pp. 655-664, 2002.

[10] X. M. Li and Z. Y. Shi, "Evaluation of roundness error from coordinate data using curvature technique," Measurement, vol. 43, no. 2, pp. 164-168, 2010.

[11] X. Wen and A. Song, "An improved genetic algorithm for planar and spatial straightness error evaluation," International Journal of Machine Tools and Manufacture, vol. 43, no. 11, pp. 1157-1162, 2003.

[12] C. H. Liu, W. Y. Jywe, and C. K. Chen, "Quality assessment on a conical taper part based on the minimum zone definition using genetic algorithms," International Journal of Machine Tools and Manufacture, vol. 44, no. 2-3, pp. 183-190, 2004.

[13] X. L. Wen, J. C. Huang, D. H. Sheng, and F. L. Wang, “Conicity and cylindricity error evaluation using particle swarm optimization," Precision Engineering, vol. 34, no. 2, pp. 338-344, 2010.

[14] X. L. Wen, Y. B. Zhao, F. L. Wang, and D. X. Wang, "Particle swarm optimization for the evaluation of cross-section contour error of variation elliptical piston skirt," in Proceedings of the International Conference on Computer Application and System Modeling, pp. V131-V135, October 2010.

[15] J. Wolfgang, "Quasi-Monte Carlo sampling to improve the efficiency of Monte Carlo EM," Computational Statistics and Data Analysis, vol. 48, no. 4, pp. 685-701, 2005.

[16] J. Kennedy and R. Eberhart, "Particle swarm optimization," in Proceedings of the IEEE International Conference on Neural Networks, pp. 1942-1948, December 1995.

[17] R. Eberhart, Y. Shi, and J. Kennedy, Swarm Intelligence, Morgan Kaufmann, San Mateo, Calif, USA, 2001.

[18] Y. del Valle, G. K. Venayagamoorthy, S. Mohagheghi, J. C. Hernandez, and R. G. Harley, "Particle swarm optimization: basic concepts, variants and applications in power systems," IEEE Transactions on Evolutionary Computation, vol. 12, no. 2, pp. 171-195, 2008.

[19] M. Richards and D. Ventura, "Choosing a starting configuration for particle swarm optimization," in Proceedings of the IEEE International Joint Conference on Neural Networks, pp. 2309-2312, July 2004.

[20] E. F. Campana, G. Fasano, and A. Pinto, "Dynamic system analysis and initial particles position in particle swarm optimization," in Proceedings of the IEEE Swarm Intelligence Symposium, pp. 202-209, May 2006.

[21] G. Lei, "Adaptive random search in quasi-Monte Carlo methods for global optimization," Computers $\mathcal{E}$ Mathematics with Applications, vol. 43, no. 6-7, pp. 747-754, 2002.

[22] H. Maaranen, K. Miettinen, and M. M. Mäkelä, "Quasi-random initial population for genetic algorithms," Computers E Mathematics with Applications, vol. 47, no. 12, pp. 1885-1895, 2004.

[23] R. C. Eberhart and Y. Shi, "Comparing inertia weights and constriction factors in particle swarm optimization," in Proceedings of the Congress on Evolutionary Computation, pp. 84-88, July 2000. 
[24] J. J. Liang, A. K. Qin, P. N. Suganthan, and S. Baskar, "Comprehensive learning particle swarm optimizer for global optimization of multimodal functions," IEEE Transactions on Evolutionary Computation, vol. 10, no. 3, pp. 281-295, 2006. 


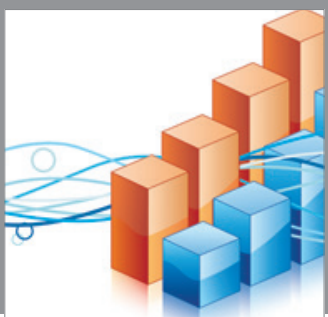

Advances in

Operations Research

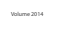

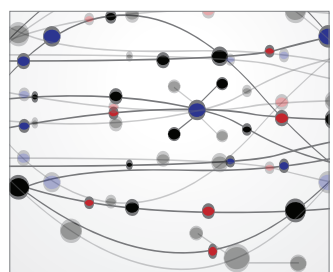

\section{The Scientific} World Journal
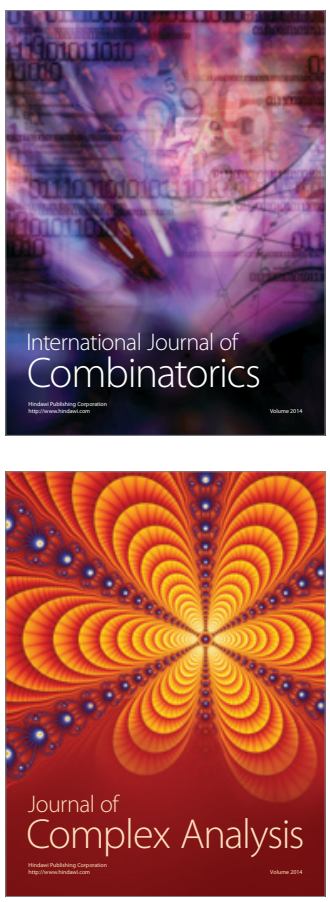

International Journal of

Mathematics and

Mathematical

Sciences
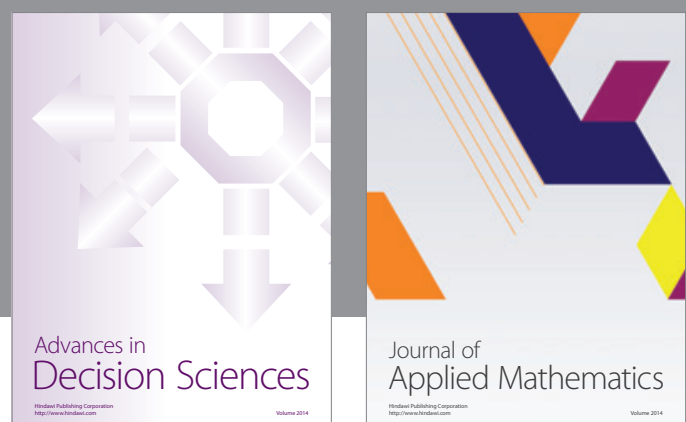

Journal of

Applied Mathematics
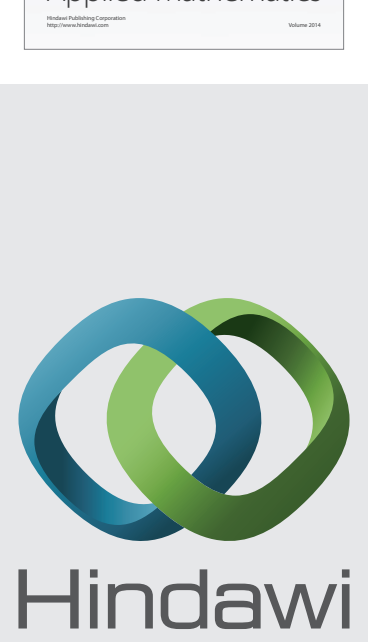

Submit your manuscripts at http://www.hindawi.com
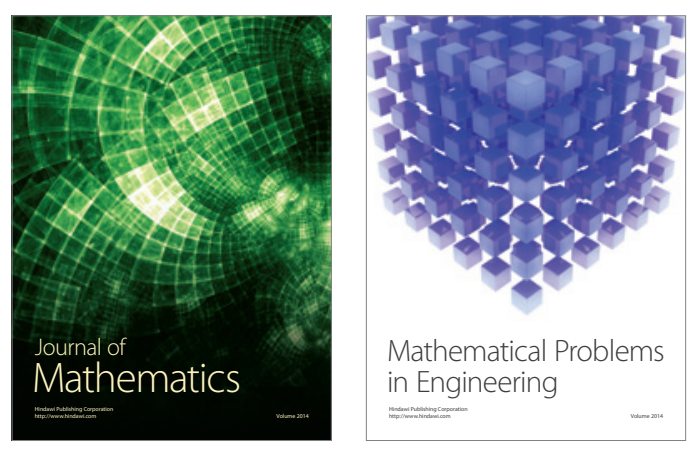

Mathematical Problems in Engineering
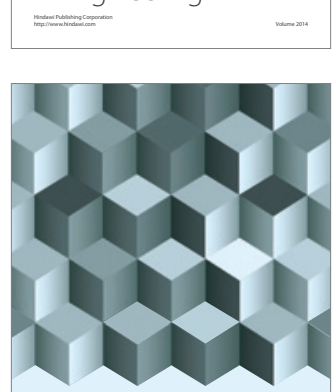

Journal of

Function Spaces
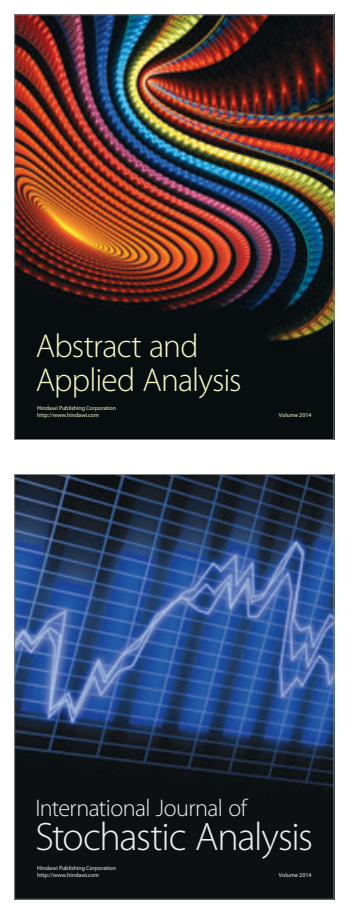

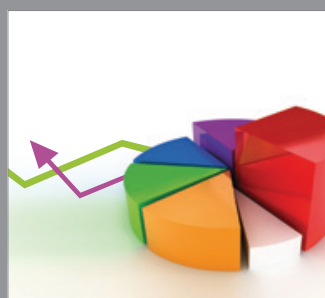

ournal of

Probability and Statistics

Promensencen
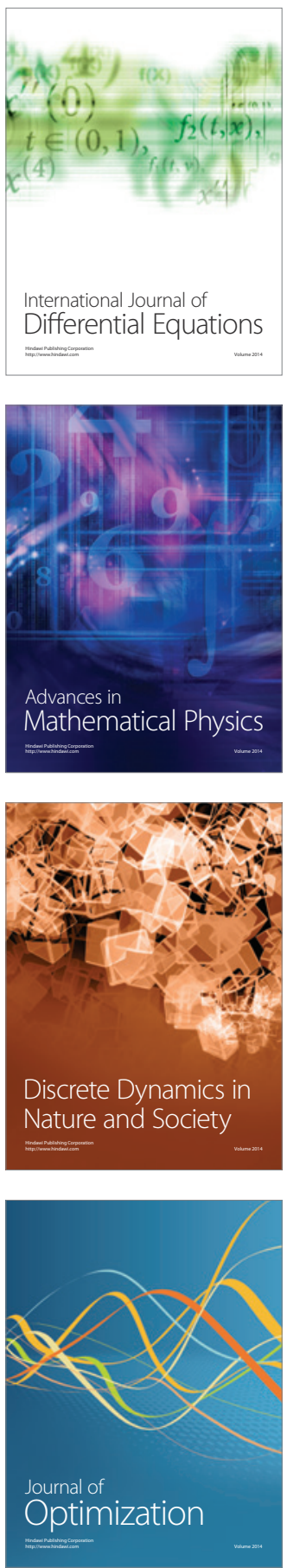